\title{
Modal Interferometer Based on ARROW Fiber for Strain and Temperature Measurement
}

\author{
S. H. Aref, O. Frazão, P. Caldas, L. A. Ferreira, F. M. Araújo, J. L. Santos, H. Latifi, P. Foy, T. Hawkins, \\ J. Ballato, T. Her, and F. Farahi
}

\begin{abstract}
In this letter, interferometric sensors based on antiresonance reflecting optical waveguide (ARROW) fibers were developed, and used to sense strain and temperature. Two types of solidcore ARROW fibers were considered and signal demodulation was achieved by using the white light interferometric technique. The ARROW fibers have two rings of high index rods arranged in a hexagonal structure with a lattice constant of $\approx 6 \mu \mathrm{m}$. The different sizes of the rods cause different measurand sensitivities for the two fibers. Resolutions of $\pm 1.1 \mu \varepsilon$ and $\pm 0.07{ }^{\circ} \mathrm{C}$ were achieved for strain and temperature, respectively.
\end{abstract}

Index Terms-Antiresonance reflecting optical waveguide (measurand sensitivities), fiber sensors, white light interferometry.

\section{INTRODUCTION}

$\mathbf{M}$ ICROSTRUCTURED optical fibers (MOFs) have a core, generally with a pure material, while the cladding is composed of a 2-D lattice arranged in a symmetric pattern. There has been considerable interest on the sensing capabilities of these fibers [1]-[8]. Several types of MOF-based sensors, such as those based on suspended core fibers [1], index-guiding fibers [2], or photonic band gap fibers [3] have been reported. The geometry of some of these fibers can be designed to yield extremely large evanescent fields [1]. On basis of this, the detection of gases that diffused into the holes of MOF has been experimentally demonstrated [2]-[4]. On the other hand, the measurement of some other physical parameters have also

Manuscript received May 14, 2009; revised July 06, 2009. First published July 31, 2009; current version published October 16, 2009. The work of J. Ballato and T. Her was supported by the Photonics Technology Access Program of Optoelectronics Industry Development Association to make the ARROW fibers.

S. H. Aref is with the Institute for Systems and Computer Engineering of Porto, 4169-007 Porto, Portugal, and also with the Laser and Plasma Research Institute, Shahid Beheshti University, Tehran 19839, Iran (e-mail: h-aref@cc. sbu.ac.ir).

O. Frazão, P. Caldas, L. A. Ferreira, and F. M. Araújo are with the Institute for Systems and Computer Engineering of Porto, 4169-007 Porto, Portugal (e-mail: h-aref@cc.sbu.ac.ir).

J. L. Santos is with the Institute for Systems and Computer Engineering of Porto, 4169-007 Porto, Portugal, and also with the Department de Física, da Faculdade de Ciências, da Universidade do Porto, 4169-007 Porto, Portugal (e-mail: h-aref@cc.sbu.ac.ir).

H. Latifi is with the Laser and Plasma Research Institute, Shahid Beheshti University, Tehran 19839, Iran.

P. Foy, T. Hawkins, and J. Ballato are with the Center for Optical Materials Science and Engineering Technologies, Clemson University, Clemson, SC 29625 USA.

T. Her and F. Farahi are with the Department of Physics and Optical Science, University of North Carolina at Charlotte, Charlotte, NC 28223 USA.

Color versions of one or more of the figures in this letter are available online at http://ieeexplore.ieee.org.

Digital Object Identifier 10.1109/LPT.2009.2028312 been reported, for example, bend and shape [5], strain and temperature [6], as well as hydrostatic pressure [7].

One particular class of MOFs is the antiresonance reflecting optical waveguide (ARROW) fibers, in which the cladding is composed of high index inclusions compared to the core material [9]. The guiding mechanism of ARROW fibers is similar to the simpler ARROW waveguides [10], in which the light is confined in the lower index core by antiresonant reflection of the high index layer. In ARROW fibers, resonant wavelengths corresponding to transmission minima can be written as [11] follows:

$$
\lambda_{r}=\frac{2 d}{m+(1 / 2)} \sqrt{n_{2}^{2}-n_{1}^{2}}
$$

where $m, n_{1}, n_{2}$, and $d$ are the resonance number $(m=$ $1,2,3, \ldots$,$) , the refractive index of the cladding material,$ the refractive index of the high-index rods, and the diameter of these rods, respectively. At these minima transmission wavelengths, the light couples strongly to the cutoff modes of the high index inclusion, and therefore, leaks out of the core [12]. In the antiresonant wavelength region, light propagates inside the core by antiresonant reflection from high-index rods. This simple analytical formula has been used to characterize some properties of these fibers and consider their application potential. For instance, a tunable filter has been designed by considering the dependency with temperature of the transmission minima wavelengths [13].

ARROW fibers are usually fabricated by filling the air holes of index-guiding photonic crystal fibers with high-index material, such as liquid crystal [14], [15] or high-index Cargille liquid [8], [9]. Another fabrication technique relies on using two different glasses, an approach mentioned as all-solid ARROW fibers [16], [17].

The sensing capabilities of ARROW fibers have been addressed in some literatures [8], [14]. Filling the air holes of index-guiding fiber with temperature or electric-field-changeable refractive index material, and observing the variation of the transmitted spectrum can be the basis for applications as filtering devices [13] or analyte sensing elements [8]. This concept was also applied to demonstrate the active control of the bandgap spectral position of photonic crystal fibers [15].

In this letter, the sensing properties of two types of solid ARROW fibers for strain and temperature measurement have been investigated. Interferometric sensors were developed and their interrogation performed using coherence addressing and pseudoheterodyne processing. 


\section{EXPERIMENTAL SETUP AND RESULTS}

The cladding and the high-index rods materials of ARROW fiber are of borosilicate and germanate glasses, respectively. The fibers have a hexagonal structure with a lattice constant of $\approx 6 \mu \mathrm{m}$. The only difference between the two types of ARROW fibers used is the rods diameter. The diameters of smaller (type 1) and bigger rods (type 2) are 2 and $3 \mu \mathrm{m}$, respectively. Rods of the germanate glass were first drawn into cane and then sleeved into the borosilicate cladding tube, which had been fabricated with the periodic arrangement of holes necessary for each ARROW fiber design. The borosilicate-germanate glass preforms were drawn at Clemson University at approximately $1000{ }^{\circ} \mathrm{C}$. The refractive index of the glass and the germanate rods are 1.46 and 1.71 , respectively. Theoretical effective refractive indexes (real and imaginary parts) of both fibers can be obtained using the boundary integral method [18].

The ARROW fiber sensing head consists of a length of an ARROW fiber connected at both ends to a single-mode fiber (SMF-28) by using a conventional fusion splice machine (Fujikura FSM-60S). As aforementioned, the ARROW fiber was made from a borosilicate glass and the softening temperature of this material is much lower than that of fused silica. Therefore, in order to have reasonable loss, the splicing procedure has been performed in the manual mode at the optimum parameters determined through a series of experiments. In particular, it was found that the best values for the electric arc intensity and duration were -100 bit and $300 \mathrm{~ms}$, respectively. Meanwhile, the arc position should be in the single-mode fiber position, some distance away from the ARROW fiber. With this strategy, the splice loss was typically $\approx 6 \mathrm{~dB}$. From the perception acquired, this loss can be reduced by following two paths. One, more practical, is to use a splicing machine that allows lower splicing arc intensities; another, more fundamental, is to enhance the overlap of the guided mode in the SMF-28 fiber with the fundamental mode of the ARROW fiber, which can be done by fabricating this fiber with tuned dimensions of the cross-section structures. The lengths of two ARROW fibers used in all experiments were $\approx 10 \mathrm{~mm}$. The broadband light, after propagation along the input SMF-28, will excite the fundamental (and also higher order modes) of the ARROW fiber. At the end of this fiber length, optical power from the ARROW modes will couple to the fundamental mode of the output SMF-28, originating an interferometric channeled spectrum pattern. Therefore, this optical configuration is a modal fiber interferometer in a Mach-Zehnder topology. In order to characterize the strain and temperature sensitivities of the sensing heads, white light interferometry has been used. Fig. 1 schematically shows the setup implemented. The light source used was a superluminescent Er-doped fiber amplified spontaneous emission (ASE) source with power, central wavelength, and full-width at half-maximum (FWHM) of $1.8 \mathrm{~mW}$, and 1550 and $60 \mathrm{~nm}$, respectively. In order to measure the sensing head phase changes, a second interferometer was built to implement coherence analysis. This interferometer is a conventional fiber Michelson interferometer with an open air path in one of its arms, which is adjustable to match the optical path difference of the sensing interferometer. The fiber in the other arm of the interferometer is wrapped around a ring-shaped piezoelectric transducer that is modulated with an electrical sawtooth waveform, with amplitude adjusted to obtain

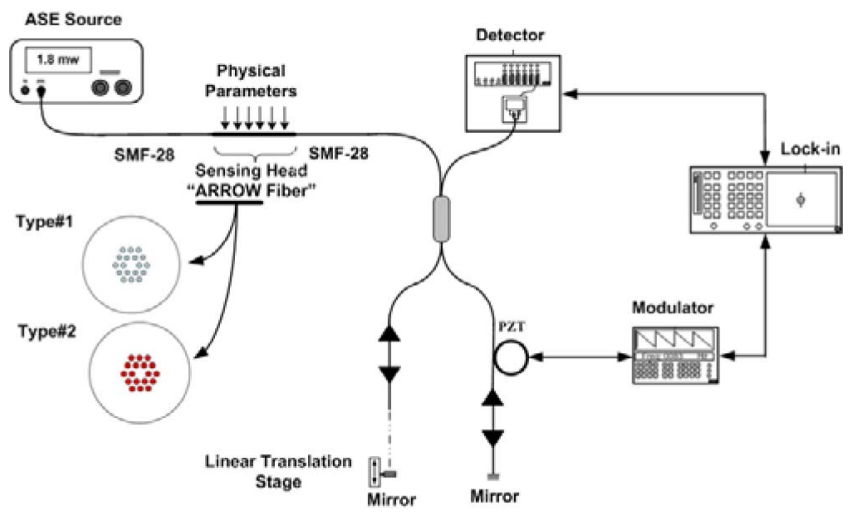

Fig. 1. Schematics of the experimental setup and cross sections of the ARROW fibers type 1 and type 2 with rods diameters of 2 and $3 \mu \mathrm{m}$, respectively.

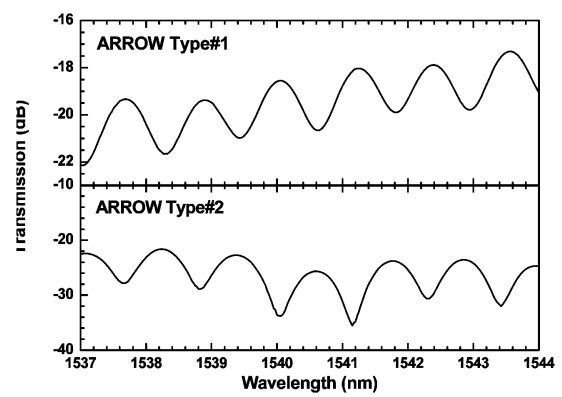

Fig. 2. Channeled spectrum of the interferometric sensing heads associated with the two types of ARROW fibers.

a signal at the photodetection suitable for pseudoheterodyne processing. After adequate electronic filtering, this signal has the form of an electric carrier $(90 \mathrm{~Hz})$, with a phase that mirrors the optical phase of the tandem interferometric system. This pseudoheterodyne processing technique is known to provide sensitive interferometric phase reading [19].

Fig. 2 shows the channeled spectra obtained for the sensing heads associated with the two ARROW fiber types. They were subjected to variations of strain and temperature. The corresponding phase changes are shown in Fig. 3. The experimental data were fitted to linear functions, resulting into the sensitivity coefficients shown in Table I. These results show a higher strain and temperature coefficients for the type-2 ARROW fiber. This means that the amount of rod material is important in setting the fiber sensitivity to these measurands. In particular, for the case of strain, it can be concluded that the fractional increase of inclusion area in the fiber cross section originates a decrease in the Young's modulus of the structure. In order to evaluate the measurand resolutions achievable with these sensing heads, the sensor output was registered during a measurand step change, as shown in Fig. 4. From the phase rms fluctuations during the periods of constant measurand values and the phase step change induced by a corresponding measurand step, it is possible to obtain the resolutions values, which are shown in Table I.

\section{CONCLUSION}

In this letter, interferometric sensors based on ARROW fibers were investigated. Two types of solid core ARROW fibers were considered (one with smaller germanate rods and the other with 

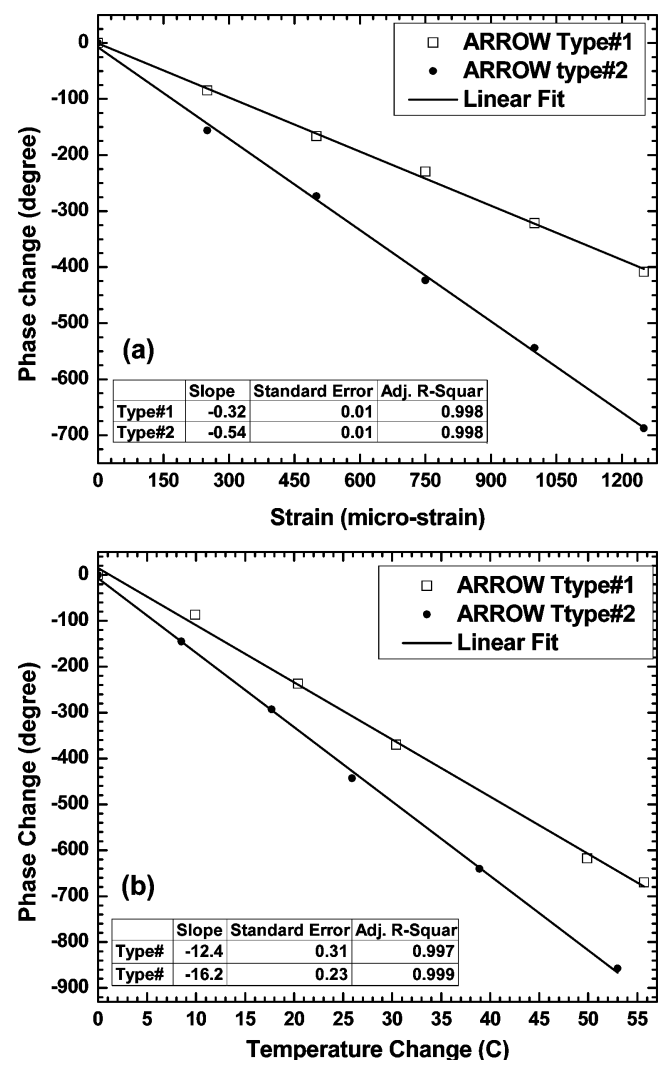

Fig. 3. Variation of the interferometric phase due to changes of: (a) applied strain and (b) temperature- the inserted lower tables show the parameters of the linear fits.

TABLE I

SENSITIVITY AND RESOLUTION VALUES OBTAINED With THE ARROW-FIBER-BASED SENSING HEADS

\begin{tabular}{ccccc}
\hline $\begin{array}{c}\text { ARROW } \\
\text { Fiber }\end{array}$ & $\begin{array}{c}\text { Strain } \\
\text { sensitivity } \\
\left(\text { degree. } \mu \varepsilon^{-1}\right)\end{array}$ & $\begin{array}{c}\text { Temperature } \\
\text { sensitivity } \\
\left(\text { degree. } .^{\circ} \mathrm{C}^{-1}\right)\end{array}$ & $\begin{array}{c}\text { Strain } \\
\text { Resolution } \\
( \pm \mu \varepsilon)\end{array}$ & $\begin{array}{c}\text { Temperature } \\
\text { Resolution } \\
\left( \pm^{\circ} \mathrm{C}\right)\end{array}$ \\
\hline Type 1 & -0.32 & -12.46 & 1.8 & 0.13 \\
Type 2 & -0.54 & -16.20 & 1.1 & 0.07 \\
\hline
\end{tabular}
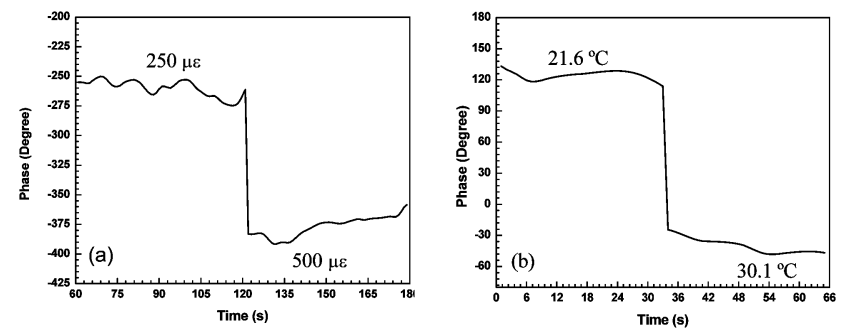

Fig. 4. Phase response of sensing head implemented with ARROW type-2 fiber for a step change in (a) strain and (b) temperature.

bigger rods in the same geometrical layout). The white light interferometric technique was used to recover the sensor interferometric phase. These structures were characterized for strain and temperature measurement. It was observed that the fiber with bigger rods allows better measurand resolutions $( \pm 1.1 \mu \varepsilon$ and $\pm 0.07{ }^{\circ} \mathrm{C}$ for strain and temperature, respectively).

\section{REFERENCES}

[1] T. M. Monro, W. Belardi, K. Furusawa, J. C. Baggett, N. G. R. Broderick, and D. J. Richardson, "Sensing with microstructured optical fibres," Meas. Sci. Technol., vol. 12, pp. 854-858, Jul. 2001.

[2] Y. L. Hoo, W. Jin, C. Shi, H. L. Ho, D. N. Wang, and S. C. Ruan, "Design and modeling of a photonic crystal fiber gas sensor," Appl. Opt., vol. 42, no. 18, pp. 3509-3515, Jun. 2003.

[3] R. Thapa, K. Knabe, K. L. Corwin, and B. R. Washburn, "Arc fusion splicing of hollow-core photonic bandgap fibers for gas-filled fiber cells," Opt. Express, vol. 14, no. 21, pp. 9576-9583, Oct. 2006.

[4] Y. L. Hoo, W. Jin, H. L. Ho, D. N. Wang, and R. S. Windeler, "Evanescent-wave gas sensing using microstructure fiber," Opt. Eng., vol. 41, no. 1, pp. 8-9, Jan. 2002.

[5] W. N. MacPherson, M. J. Gander, R. McBride, J. D. C. Jones, P. M. Blanchard, J. G. Burnett, A. H. Greenaway, B. Mangan, T. A. Birks, J. C. Knight, and P. S. J. Russell, "Remotely addressed optical fibre curvature sensor using multicore photonic crystal fibre," Opt. Commun., vol. 193, no. 1-6, pp. 97-104, Jun. 2001.

[6] C. Zhao, L. Xiao, J. Ju, M. S. Demokan, and W. Jin, "Strain and temperature characteristics of a long-period grating written in a photonic crystal fiber and its application as a temperature-insensitive strain sensor," J. Lightw. Techol., vol. 26, no. 2, pp. 220-227, Jan. 15, 2008.

[7] W. N. MacPherson, E. J. Rigg, J. D. C. Jones, V. V. Ravi, K. Kumar, J. C. Knight, and P. S. J. Russell, "Finite-element analysis and experimental results for a microstructured fibre with enhanced hydrostatic pressure sensitivity," J. Lightw. Technol., vol. 23, no. 3, pp. 1227-1231, Mar. 2005.

[8] N. M. Litchinitser and E. Poliakov, "Antiresonant guiding microstructured optical fibers for sensing applications," Appl. Phys. B, Lasers Opt., vol. 81, no. 2-3, pp. 347-351, Jul. 2005.

[9] R. T. Bise, R. S. Windeler, K. S. Kranz, C. Kerbage, B. J. Eggleton, and D. J. Trevor, "Tunable photonic bandgap fiber," in Opt. Fiber Commun. Conf., Washington, DC, 2002, vol. 70, OSA trends in Optics and photonic series, pp. 466-468.

[10] M. A. Duguay, Y. Kukubun, T. L. Koch, and L. Pfeiffer, "Antiresonant reflecting optical waveguides in $\mathrm{SiO}_{2}$-Si multiplayer structures," Appl. Phys. Lett., vol. 49, pp. 13-15, Jul. 1986.

[11] N. M. Litchinitser, S. C. Dunn, B. Usner, B. J. Eggleton, T. P. White, R. C. McPhedran, and C. M. Sterke, "Resonances in microstructured optical waveguides," Opt. Express, vol. 11, no. 10, pp. 1243-1251, May 2003.

[12] T. P. White, R. C. McPhedran, C. M. der Sterke, N. M. Litchinitser, and B. J. Eggleton, "Resonance and scattering in microstructured optical fibers," Opt. Lett., vol. 27, no. 22, pp. 1977-79, Nov. 2002.

[13] N. Litchinitser, S. Dunn, P. Steinvurzel, B. Eggleton, T. White, R. McPhedran, and C. der Sterke, "Application of an arrow model for designing tunable photonic devices," Opt. Express, vol. 12, no. 8, pp. 1540-1550, Apr. 2004.

[14] T. R. Wolinski, A. Czapla, S. Ertman, M. Tefelska, A. W. Domanski, J. Wójcik, E. Nowinowski-Kruszelnicki, and R. Dabrowski, "Photonic liquid crystal fibers for sensing applications," IEEE Trans. Instrum. Meas., vol. 57, no. 8, pp. 1796-1802, Aug. 2008.

[15] T. Larsen, A. Bjarklev, D. Hermann, and J. Broeng, "Optical devices based on liquid crystal photonic bandgap fibres," Opt. Express, vol. 11, no. 20, pp. 2589-2596, Oct. 2003.

[16] A. Argyros, T. A. Birks, S. G. Leon-Saval, C. M. B. Cordeiro, and P. S. J. Russell, "Guidance properties of low-contrast photonic bandgap fibres," Opt. Express, vol. 32, no. 7, pp. 2503-2511, Apr. 2005.

[17] G. Ren, P. Shum, L. Zhang, X. Yu, W. Tong, and J. Luo, "Low-loss allsolid photonic bandgap fiber," Opt. Lett., vol. 32, no. 9, pp. 1023-1025, May 2007.

[18] M. Cho, W. Cai, and T. Her, "A boundary integral equation method for photonic crystal fibers," J. Sci. Comput., vol. 28, no. 2/3, pp. 263-278, Sep. 2006.

[19] P. Caldas, P. A. S. Jorge, F. M. Araujo, L. A. Ferreira, M. B. Marques, G. Rego, and J. L. Santos, "Fiber modal Michelson interferometers with coherence addressing and heterodyne interrogation," Opt. Eng., vol. 47, no. 4, p. 44401, Apr. 2008. 